\title{
On the rank of incidence matrices in projective Hjelmslev spaces
}

\author{
Ivan Landjev • Peter Vandendriesche
}

Received: 12 August 2013 / Revised: 11 February 2014 / Accepted: 6 March 2014 /

Published online: 30 March 2014

(C) Springer Science+Business Media New York 2014

\begin{abstract}
Let $R$ be a finite chain ring with $|R|=q^{m}, R / \operatorname{Rad} R \cong \mathbb{F}_{q}$, and let $\Omega=$ $\operatorname{PHG}\left({ }_{R} R^{n}\right)$. Let $\tau=\left(\tau_{1}, \ldots, \tau_{n}\right)$ be an integer sequence satisfying $m=\tau_{1} \geq \tau_{2} \geq \cdots \geq$ $\tau_{n} \geq 0$. We consider the incidence matrix of all shape $\boldsymbol{m}^{s}=(\underbrace{m, \ldots, m}_{s})$ versus all shape $\tau$ subspaces of $\Omega$ with $\boldsymbol{m}^{s} \preceq \tau \preceq \boldsymbol{m}^{n-s}$. We prove that the rank of $M_{\boldsymbol{m}^{s}, \tau}(\Omega)$ over $\mathbb{Q}$ is equal to the number of shape $\boldsymbol{m}^{s}$ subspaces. This is a partial analog of Kantor's result about the rank of the incidence matrix of all $s$ dimensional versus all $t$ dimensional subspaces of $\mathrm{PG}(n, q)$. We construct an example for shapes $\sigma$ and $\tau$ for which the rank of $M_{\sigma, \tau}(\Omega)$ is not maximal.
\end{abstract}

Keywords Projective Hjelmslev spaces - Finite chain rings - Modules over finite chain rings

Mathematics Subject Classification $51 \mathrm{C} 05 \cdot 51 \mathrm{E} 05 \cdot 51 \mathrm{E} 23$

\section{Preliminaries}

It is known that the rank of an incidence matrix of all $s$-dimensional versus all $t$-dimensional subspaces in $\operatorname{PG}(n, q), 0 \leq s<t \leq n-s-1$, is equal to the number of the $s$-spaces in the geometry [6]. In this paper, we prove a similar result for the incidence matrices of shape $\sigma$ versus shape $\tau$ subspaces in the projective Hjelmslev space $\operatorname{PHG}\left({ }_{R} R^{n}\right)$, where $R$ is a chain

This is one of several papers published in Designs, Codes and Cryptography comprising the "Special Issue on Coding and Cryptography".

I. Landjev (ه)

New Bulgarian University, Montevideo str. 21, 1618 Sofia, Bulgaria

e-mail: i.landjev@nbu.bg

P. Vandendriesche

Department of Mathematics (WE01), Ghent University, Krijgslaan 281-S22, 9000 Ghent, Belgium

e-mail: pv@cage.ugent.be 
ring. We put some natural restrictions on the shapes $\sigma$ and $\tau$. For instance, in order to avoid trivial matrices, we require that $\sigma \preceq \tau$ in the partial order of the shapes of all subspaces in $\operatorname{PHG}\left({ }_{R} R^{n}\right)$.

We start with some definitions and results about chain rings and modules over finite chain rings. Let $R$ be a finite chain ring with $|R|=q^{m}, R / \operatorname{Rad} R \cong \mathbb{F}_{q}$, where $q=p^{h}$ with $p$ prime. It is known that all non-trivial ideals of $R$ have the form $R \theta^{i}=\theta^{i} R$ for some $\theta \in \operatorname{Rad} R \backslash(\operatorname{Rad} R)^{2}$ and some $i \in\{1, \ldots, m-1\}$. Denote by $\Gamma=\left\{\gamma_{0}=0, \gamma_{1}=\right.$ $\left.1, \gamma_{2}, \ldots, \gamma_{q-1}\right\}$ a set of elements of $R$ no two of which are congruent $\operatorname{modulo} \operatorname{Rad} R$. Every element $r \in R$ can be written uniquely as $r=\sum_{i=0}^{m-1} r_{i} \theta^{i}$, where $r_{i} \in \Gamma$. Throughout the paper the letters $m, p, q, h, \theta, \Gamma, \gamma_{i}$ will keep the meaning fixed above. For the basic properties of chain rings we refer to $[2,9,10]$.

The structure of finitely generated modules over the finite chain rings $R$ is well-known. In this note we shall be confined to left modules. This is no restriction since every left module can be considered as a right module over the opposite chain ring. For every left finitely generated module ${ }_{R} M$ over the chain ring $R$ there exists a unique partition of $\log _{q}|M|$ into parts $\lambda_{i} \leq m$, denoted $\lambda=\left(\lambda_{1}, \ldots, \lambda_{k}\right) \vdash \log _{q}|M|$, such that

$$
{ }_{R} M \cong R /(\operatorname{Rad} R)^{\lambda_{1}} \oplus \cdots \oplus R /(\operatorname{Rad} R)^{\lambda_{k}} .
$$

The partition $\lambda$ is called the shape and the integer $k$ is called the rank of ${ }_{R} M$. The conjugate partition $\lambda^{\prime}=\left(\lambda_{1}^{\prime}, \lambda_{2}^{\prime} \ldots\right) \vdash \log _{q}|M|$, where $\lambda_{i}^{\prime}$ is the number of the parts in $\lambda$ greater or equal to $i$, is referred to as the conjugate shape of ${ }_{R} M$.

For a given positive integer $n$ and a non-increasing sequence of non-negative integers $\kappa=\left(\kappa_{1}, \ldots, \kappa_{n}\right)$ we denote by $\mathcal{G}(n, \kappa)$ the set of all submodules of shape $\kappa$ of ${ }_{R} R^{n}$. In what follows, we denote the sequence $(\underbrace{m, \ldots, m}_{k}, \underbrace{0, \ldots, 0}_{n-k})$ by $\boldsymbol{m}^{k}$. For two sequences $\lambda=$ $\left(\lambda_{1}, \ldots, \lambda_{n}\right)$ and $\mu=\left(\mu_{1}, \ldots, \mu_{n}\right)$, we write $\lambda \preceq \mu$ iff $\lambda_{i} \leq \mu_{i}$ for all $i=1, \ldots, n$.

We shall need formulae for the number of the submodules of given shape $\mu$ contained in a fixed module of shape $\lambda$ over a finite chain ring $R$. For the special case of $R=\mathbb{Z}_{q^{m}}$ they are known from [1]. The case of a general chain ring $R$ is settled in [8, Chapter 2].

Theorem 1 [8] Let ${ }_{R} M$ be a module of shape $\lambda$. For every partition $\mu$ satisfying $\mu \leq \lambda$ the module ${ }_{R} M$ has exactly

$$
\left[\begin{array}{l}
\lambda \\
\mu
\end{array}\right]_{q}:=\prod_{i=1}^{m} q^{\mu_{i+1}^{\prime}\left(\lambda_{i}^{\prime}-\mu_{i}^{\prime}\right)} \cdot\left[\begin{array}{l}
\lambda_{i}^{\prime}-\mu_{i+1}^{\prime} \\
\mu_{i}^{\prime}-\mu_{i+1}^{\prime}
\end{array}\right]_{q}
$$

submodules of shape $\mu$. By convention, $\lambda_{m+1}^{\prime}=\mu_{m+1}^{\prime}=0$.

The symbol $\left[\begin{array}{l}n \\ k\end{array}\right]_{q}$ with $k \leq n$ integers and $q$ a prime power, denotes the classical Gaussian coefficient:

$$
\left[\begin{array}{l}
n \\
k
\end{array}\right]_{q}=\frac{\left(q^{n}-1\right) \ldots\left(q^{n-k+1}-1\right)}{\left(q^{k}-1\right) \ldots(q-1)} .
$$

By duality, Theorem 1 allows to find the number of shape $\lambda$ submodules of ${ }_{R} R^{n}$ containing a fixed submodule of shape $\mu$. One has to apply Theorem 1 to the dual submodules that have shapes $\boldsymbol{m}^{n}-\lambda$ and $\boldsymbol{m}^{n}-\mu$, respectively. So, this number equals $\left[\begin{array}{c}\boldsymbol{m}^{n}-\mu \\ \boldsymbol{m}^{n}-\lambda\end{array}\right]_{q}$.

The (left) projective Hjelmslev geometries $\operatorname{PHG}\left({ }_{R} R^{n}\right)$ are produced from the finite modules ${ }_{R} R^{n}$ in the same way one produces the classical projective geometries $\operatorname{PG}(n-1, q)$ from the vector spaces $\mathbb{F}_{q}^{n}$. The geometry $\left.\operatorname{PHG}_{(} R^{n}\right)$ is defined as an incidence structure 
$(\mathcal{P}, \mathcal{L}, I)$ having as points the free rank 1 submodules of ${ }_{R} R^{n}$ and as lines the free rank 2 submodules of $R_{R} R^{n}$. Incidence is given by set-theoretical inclusion. The set of points contained in a submodule ${ }_{R} M \subset_{R} R^{n}$ which is of shape $\lambda$ is called a subspace of shape $\lambda$. The subspaces defined by free submodules of ${ }_{R} R^{n}$ are called Hjelmslev subspaces.

Two subspaces $\boldsymbol{L}$ and $\boldsymbol{M}$ of the same shape defined by the modules ${ }_{R} L$ and ${ }_{R} M$, respectively, are called $i$-neighbors if ${ }_{R} L={ }_{R} M+\theta^{i} R^{n}$. This fact is denoted by $L \alpha_{i} M$. It can be checked that $i$-neighborhood is an equivalence relation on the set of all subspaces. The equivalence class of all subspaces that are $i$-neighbors to $\boldsymbol{L}$ is denoted by $[\boldsymbol{L}]^{(i)}$. Set

$$
\mathcal{P}^{(i)}=\left\{[x]^{(i)} \mid x \in \mathcal{P}\right\}, \mathcal{L}^{(i)}=\left\{[L]^{(i)} \mid L \in \mathcal{L}\right\},
$$

and

$$
I^{(i)}=\left\{\left([x]^{(i)},[L]^{(i)}\right) \mid \exists x^{\prime} \in[x]^{(i)}, L^{\prime} \in[L]^{(i)}:\left(x^{\prime}, L^{\prime}\right) \in I\right\} .
$$

Theorem 2 [3-5] The incidence structure $\left(\mathcal{P}^{(i)}, \mathcal{L}^{(i)}, I^{(i)}\right)$ is isomorphic to the geometry PHG $\left(R / \theta^{i} R\left(R / \theta^{i} R\right)^{n}\right)$.

Let us note that $R / \theta^{i} R$ is again a chain ring of size $q^{i}$, with residue field $\mathbb{F}_{q}$. In particular, for $i=1$ we get $\left(\mathcal{P}^{(1)}, \mathcal{L}^{(1)}, I^{(1)}\right) \cong \mathrm{PG}(n-1, q)$.

Another family of substructures $\operatorname{PHG}\left({ }_{R} R^{n}\right)$ is given by the next theorem. Fix a $(k-1)$ dimensional Hjelmslev subspace $\boldsymbol{M}$ in $\operatorname{PHG}\left({ }_{R} R^{n}\right)$, and an integer $j$ with $0<j<m$. Denote by $\mathcal{P}_{j}(\boldsymbol{M})$ (resp. $\mathcal{L}_{j}(\boldsymbol{M})$ ) the set of all points (resp. lines) that have an $j$-th neighbour on $\boldsymbol{M}$. Let $[x]^{(m-j)}, x \in \mathcal{P}_{j}(\boldsymbol{M})$, be the neighbour class of all $(m-j)$-th neighbors to $x$, and, similarly, let $[L]^{(m-j)}, L \in \mathcal{L}_{j}(\boldsymbol{M})$, be the neighbour class of all $(m-j)$-th neighbors to $L$, Define a new point set

and a new set of lines $\mathfrak{L}$ as

$$
\mathfrak{P}=\left\{\boldsymbol{L} \cap[L]^{(m-j)} \mid L \in \mathcal{P}_{j}(\boldsymbol{M}), \boldsymbol{L} \propto_{i} \boldsymbol{M}, \boldsymbol{L} \cap[x]^{(m-j)} \neq \varnothing\right\} .
$$

$$
\mathfrak{L}=\left\{\boldsymbol{L} \cap[L]^{(m-j)} \mid L \in \mathcal{L}_{j}(\boldsymbol{M}), \boldsymbol{L} \boldsymbol{\alpha}_{i} \boldsymbol{M}, \boldsymbol{L} \cap[L]^{(m-j)} \neq \varnothing\right\} .
$$

The incidence $\mathfrak{J} \subseteq \mathfrak{P} \times \mathfrak{L}$ is given by set-theoretical inclusion.

Theorem 3 [3-5] The incidence structure (P, $\mathfrak{L}, \mathfrak{J})$ can be embedded isomorphically into $\left.\operatorname{PHG}_{R /(\operatorname{Rad} R)^{m-j}}\left(R /(\operatorname{Rad} R)^{m-j}\right)^{n}\right)$. The missing part consists of the points of an $(n-k-$ 1)-dimensional Hjelmslev subspace $\boldsymbol{H}$ and all the subspaces which have commom points with $\boldsymbol{H}$.

Note that $R /(\operatorname{Rad} R)^{i}$ is again a chain ring with $q^{i}$ elements. In the special case when $j=1$, the structure $(\mathfrak{P}, \mathfrak{L}, \mathfrak{J})$ is a part of $\operatorname{PHG}\left({ }_{S} S^{n}\right)$ where $S$ is a chain ring with $q^{m-1}$ elements. In this case, subspaces of shape $\sigma=\left(\sigma_{1}, \ldots, \sigma_{t}\right)$ (we suppress trailing zeros) in $[\boldsymbol{M}]$ become subspaces of shape $\left(\sigma_{1}-1, \ldots, \sigma_{t}-1\right)$ in $(\mathfrak{P}, \mathfrak{L}, \mathfrak{J})$.

If $\boldsymbol{M}$ is a point, the missing part is a hyperplane. Thus the $j$-th neighbour classes of points carry the structure of an affine geometry over $R /(\operatorname{Rad} R)^{j}$. For a more detailed introduction into projective Hjelmslev geometries we refer to [3-5].

\section{Incidence matrices and the main theorem}

Let $R$ be a finite chain ring with $|R|=q^{m}, R / \operatorname{Rad} R \cong \mathbb{F}_{q}$, and let $\Omega=\operatorname{PHG}\left({ }_{R} R^{n}\right)$. Let further $\sigma=\left(\sigma_{1}, \ldots, \sigma_{n}\right)$ and $\tau=\left(\tau_{1}, \ldots, \tau_{n}\right)$ be non-increasing sequences of non-negative 
integers, i.e. $m \geq \sigma_{1} \geq \cdots \geq \sigma_{n} \geq 0, m \geq \tau_{1} \geq \cdots \geq \tau_{n} \geq 0$, with $\sigma \preceq \tau$. We define a $(0,1)$-matrix $M_{\sigma, \tau}$ in which the rows are indexed by the elements $\mathcal{G}(n, \sigma)$ and columns are indexed by the elements of $\mathcal{G}(n, \tau)$. The element $m(S, T)$ which is in the row indexed by $S \in \mathcal{G}(n, \sigma)$ and the column indexed by $T \in \mathcal{G}(n, \tau)$ is defined by

$$
m(S, T)=\left\{\begin{array}{l}
1 \text { if } S \subset T, \\
0 \text { if } S \not \subset T .
\end{array}\right.
$$

We denote by $\rho(S)$ the row of $M_{\sigma, \tau}(\Omega)$ indexed by the shape $\sigma$ subspace $S$. Our goal is to prove the following theorem which is an analog of Kantor's result [6] about the rank of the incidence matrix of dimension $s$ versus dimension $t$ subspaces in $\operatorname{PG}(n-1, q)$. Since our proof relies on Kantor's theorem, we state it explicitly below.

Theorem 4 [6] Let $0 \leq s<t \leq n-s-1$ and let $M_{s, t}$ be an incidence matrix of all $s$-spaces $v$ s. all $t$-spaces of $\mathrm{PG}(n, q)$ or $A G(n, q)$. Then the rank of $M_{s, t}$ is the number of s-spaces in the geometry.

The goal of this paper is to prove the following analog of Kantor's result.

Theorem 5 (Main Theorem) Let $R$ be a finite chain ring with $|R|=q^{m}, R / \operatorname{Rad} R \cong \mathbb{F}_{q}$, and let $\Omega=\operatorname{PHG}\left({ }_{R} R^{n}\right)$ Let $\tau=\left(\tau_{1}, \ldots, \tau_{n}\right)$ be an integer sequence with

$$
m=\tau_{1} \geq \tau_{2} \geq \cdots \geq \tau_{n} \geq 0 .
$$

and with $\boldsymbol{m}^{s} \preceq \tau \preceq \boldsymbol{m}^{n-s}$. Then the rank of $M_{\boldsymbol{m}^{s}, \tau}(\Omega)$ is equal to the number of the $(s-1)$ dimensional Hjelmslev subspaces of $\Omega$, i.e. $\left[\begin{array}{c}m^{n} \\ m^{s}\end{array}\right]_{q}$.

This theorem covers the case where the rows of $M_{\sigma, \tau}(\Omega)$ are indexed by free submodules. In the last section, we construct an example of an incidence matrix $M_{\sigma, \tau}(\Omega)$ with $\sigma \neq \boldsymbol{m}^{s}$ (i.e. the subspaces of shape $\sigma$ are not Hjelmslev subspaces) which is not of full rank over $\mathbb{Q}$.

\section{A special case}

Before we start with the proof of Theorem 5, we mention briefly the case of incidence matrices with rows indexed by the points and columns indexed by the subspaces of shape $\tau$. This is a special case of Theorem 5 . The proof of Theorem 5 does not rely on this special case, but we include it because it uses the calculation of a special determinant which does not seem to be that well-known.

Let $\tau=\left(\tau_{1}, \ldots, \tau_{n}\right)$ be a non-increasing sequence of non-negative integers, i.e.

$$
m=\tau_{1} \geq \cdots \geq \tau_{n} \geq 0,
$$

with $\tau \preceq \boldsymbol{m}^{n-1}$. Given a linear order on the points and on the subspaces of shape $\tau$, we define $M(\tau)=M_{\boldsymbol{m}^{1}, \tau}=\left(m_{i j}\right)$.

The size of $M(\tau)$ is $\left[\begin{array}{c}m^{n} \\ m^{1}\end{array}\right]_{q} \times\left[\begin{array}{c}m^{n} \\ \tau\end{array}\right]_{q}$. We shall fix a particular ordering on the points of $\operatorname{PHG}\left({ }_{R} R^{n}\right)$. First we order linearly the 1 -neighbour classes of points, i.e. the elements of $\mathcal{P}^{(1)}$; further we order linearly the 2-neighbour classes of points within each 1-neighbour class. We go on in the same way until we reach a linear order of the elements of $\mathcal{P}^{(m)}$ (which are single points) within each $(m-1)$-neighbour class of points. If our indices start from 0 , i.e. our points are $x_{0}, x_{1}, \ldots$, then the points $x_{i}$ and $x_{j}$ are $k$-th neighbours iff

$$
\left\lfloor\frac{i}{q^{(m-k)(n-1)}}\right\rfloor=\left\lfloor\frac{j}{q^{(m-k)(n-1)}}\right\rfloor .
$$


Set

$$
A:=M(\tau) \cdot M^{t}(\tau) .
$$

The matrix $A=\left(a_{i j}\right)$ is a symmetric matrix of order $\left[\begin{array}{c}m^{n} \\ m^{1}\end{array}\right]_{q}$ and has in position $(i, j)$ the number of shape $\tau$ subspaces containing the points $x_{i}$ and $x_{j}$.

Let $x$ and $y$ be two points in $\operatorname{PHG}\left({ }_{R} R^{n}\right)$ with $x_{k} y$. Denote by $N_{k}$ the number of subspaces of PHG $\left({ }_{R} R^{n}\right)$ of shape $\tau=\left(\tau_{1}, \ldots, \tau_{n}\right)$ containing $x$ and $y$. Since the module $\langle x, y\rangle$ has shape $(m, m-k)$, we have $N_{k}=0$ if $\tau_{2}<m-k$ and $N_{k}>0$ if $\tau_{2} \geq m-k$. So, if $k$ is the maximal integer for which (3) is satisfied then $a_{i j}=N_{k}$. Note that $N_{m} \neq 0$ and that $N_{m}>N_{m-1}>\cdots>N_{0}>0$ or $N_{m}>N_{m-1}>\cdots>N_{k}=\cdots=N_{0}=0$ for some $k \in\{0, \ldots, m-1\}$. The inequalities above are obtained using the remark after Theorem 2 .

We need the following lemma which can be found in [7].

Lemma 1 Let $n$ be a positive integer, let $k_{0}, k_{1}, \ldots, k_{n}$ be positive integers with $k_{0}=1$, $k_{1}\left|k_{2}, \ldots, k_{n-1}\right| k_{n}$. Let $b_{0}, b_{1}, \ldots, b_{n}$ be arbitrary elements of a field $F$ and let $C$ be the $k_{n} \times k_{n}$ matrix over $F$ given by $c_{i j}=b_{\min }\left\{t:\left[\frac{i}{k_{t}}\right\rfloor=\left\lfloor\frac{j}{k_{t}}\right\rfloor\right\}$, where the rows and columns are labeled from 0 up to $k_{n}-1$. Then

$$
\operatorname{det}(C)=\prod_{i=0}^{n}\left(\sum_{j=0}^{i} k_{j}\left(b_{j}-b_{j+1}\right)\right)^{\frac{k_{n}}{k_{i}}-\frac{k_{n}}{k_{i+1}}},
$$

where by convention $b_{n+1}=0$ and $k_{n+1}=+\infty$.

Theorem 6 Let $\tau=\left(\tau_{1}, \ldots, \tau_{n}\right)$ be a non-increasing sequence of non-negative integer with $\boldsymbol{m}^{1} \preceq \tau \preceq \boldsymbol{m}^{n-1}$. Then the matrix $M(\tau)$ is of full rank over $\mathbb{R}$.

Proof For the matrix $A=M(\tau) \cdot M(\tau)^{t}$ one has $k_{i}=q^{i}$ for $i=0, \ldots, m-1, k_{m}=\left[\begin{array}{c}m^{n} \\ m^{1}\end{array}\right]_{q}$ and $b_{i}=N_{m-i}$. Now, by Lemma 1 ,

$$
\operatorname{det} A=\operatorname{det} M(\tau) M^{t}(\tau) \neq 0
$$

This implies that $A$ is of $\operatorname{rank}\left[\begin{array}{c}m^{n} \\ m^{1}\end{array}\right]_{q}$ which in turn gives that $M(\tau)$ is non-singular and its rank is equal to the number of its rows, i.e. $\left[\begin{array}{c}m^{n} \\ m^{1}\end{array}\right]_{q}$.

\section{The proof of Theorem 5}

We start with the case when the rows and the columns of $M_{\sigma, \tau}$ are indexed by Hjelmslev subspaces, i.e. $\sigma=\boldsymbol{m}^{s}$ and $\tau=\boldsymbol{m}^{t}$.

Theorem 7 Let $R$ be a chain ring with $|R|=q^{m}, R / \operatorname{Rad} R \cong \mathbb{F}_{q}$, and let $\Omega=\operatorname{PHG}\left({ }_{R} R^{n}\right)$. Let further $s$ and $t$ be integers with $1 \leq s \leq t \leq n-s$. Then the rank of $M_{\boldsymbol{m}^{s}, \boldsymbol{m}^{t}}(\Omega)$ is equal to the number of free Hjelmslev subspaces of $\Omega$ of dimension $s-1$ i.e. the rank is equal to $\left[\begin{array}{c}\boldsymbol{m}^{n} \\ \boldsymbol{m}^{s}\end{array}\right]_{q}$.

Proof We use induction on $m$. The case $m=1$ is Kantor's Theorem. Let us assume that the result is proved for all incidence matrices $M_{\boldsymbol{m}^{s}, \boldsymbol{m}^{t}}\left(\Omega^{\prime}\right)$ where $\Omega^{\prime}$ is an $(n-1)$-dimensional projective Hjelmslev geometry over a chain ring of nilpotency index at most $m-1$.

Now let $R$ be a chain ring with $|R|=q^{m}, q=p^{h}, R / \operatorname{Rad} R \cong \mathbb{F}_{q}$, and denote $\Omega=$ $\operatorname{PHG}\left({ }_{R} R^{n}\right)$. Consider two $(m-1)$-neighbor classes of Hjelmslev subspaces of shape $\boldsymbol{m}^{s}$ 
and $\boldsymbol{m}^{t}$, say $[S]^{(m-1)}=\left\{S_{1}, \ldots, S_{u}\right\}$ and $[T]^{(m-1)}=\left\{T_{1}, \ldots, T_{v}\right\}$, respectively. If some subspace from $[S]^{(m-1)}$ contains a point which is not incident with a subspace from $[T]^{(m-1)}$ then $S_{i} \not \subset T_{j}$ for any $i \in\{1, \ldots, u\}$ and any $j \in\{1, \ldots, v\}$. Hence the $u \times v$ submatrix of $M_{\boldsymbol{m}^{s}, \boldsymbol{m}^{t}}(\Omega)$ defined by the rows indexed by $S_{1}, \ldots, S_{u}$ and the columns indexed by $T_{1}, \ldots, T_{v}$ is the all-zero matrix. Otherwise, each subspace of $[S]^{(m-1)}$ is contained in the same number of subspaces from $[T]^{(m-1)}$ and each subspace from $[T]^{(m-1)}$ contains the same number of subspaces from $S^{(m-1)}$. Hence the submatrix of $M_{\boldsymbol{m}^{s}, \boldsymbol{m}^{t}}(\Omega)$ with rows indexed by the subspaces from $[S]^{(m-1)}$ and the columns indexed by the subspaces from $[T]^{(m-1)}$ is a $(0,1)$ matrix, $B$ say, with constant row and column sums. For a suitable ordering of all Hjelmslev subspaces of dimension $s-1$, resp. $t-1$, the matrix $M_{\boldsymbol{m}^{s}, \boldsymbol{m}^{t}}(\Omega)$ can be represented in the following block form:

$$
M_{\boldsymbol{m}^{s}, \boldsymbol{m}^{t}}(\Omega)=\left(A_{i, j}\right),
$$

where $i=1, \ldots, x, j=1, \ldots, y$. Here $x$ and $y$ are the numbers of the $(m-1)$-st neighbor classes of subspaces of dimension $s-1$, resp. $t-1$. By Theorem 2, we get

$$
x=q^{s(n-s)(m-2)}\left[\begin{array}{l}
n \\
s
\end{array}\right]_{q}, y=q^{t(n-t)(m-2)}\left[\begin{array}{l}
n \\
t
\end{array}\right]_{q} .
$$

If the $i$-th $(m-1)$-neighbor class of $s-1$ dimensional Hjelmslev subspaces is contained in the $j$-th $(m-1)$-neighbor class of $(t-1)$-dimensional Hjelmslev subspaces in the factor geometry then $A_{i, j}$ is a $u \times v$ matrix of zeros and ones which has the form $P B Q$ for some suitable permutation matrices $P$ and $Q$ of orders $u$ and $v$, respectively. Otherwise $A_{i, j}$ is the zero matrix. Moreover, the matrix $A=\left(a_{i, j}\right)$ of size $x \times y$ defined by

$$
a_{i, j}=\left\{\begin{array}{l}
1 \text { if } A_{i, j} \neq \mathbf{0}_{u \times v}, \\
0 \text { if } A_{i, j}=\mathbf{0}_{u \times v}
\end{array}\right.
$$

is equivalent to the incidence matrix of free $(s-1)$-dimensional versus free $(t-1)$ dimensional Hjelmslev suspaces in the factor geometry $\left.\mathrm{PHG}_{R / R \theta}(R / R \theta)^{n}\right)$. Since $R / R \theta$ has nilpotency index $m-1$, the rank of $A$ is equal to the number of its rows, by the induction hypothesis.

Assume there exists a non-trivial linear combination of the rows of the matrix $M_{\boldsymbol{m}^{s}, \boldsymbol{m}^{t}}(\Omega)$.

$$
\sum_{S} a(S) \rho(S)=\sum_{[S]^{(m-1)}} \sum_{L \in[S]^{(m-1)}} a(L) \rho(L)=\mathbf{0}
$$

where $a(L)$ are rational numbers not all zero. Define

$$
G=\left\{I+C \theta^{m-1} \mid C \text { is an } n \times n \text { matrix over } \Gamma \text { with } 0 \text { 's on the main diagonal }\right\} .
$$

$G$ is a commutative group under matrix multiplication. $G$ fixes all $(m-1)$-neighbor classes of points setwise and acts transitively on the points within these classes. Hence the orbits of $G$ on the set of all Hjelmslev subspaces are the $(m-1)$-neighbor classes of Hjelmslev spaces themselves. In particular, this is true for all $(s-1)$-dimensional Hjelmslev subspaces. Thus for every $(s-1)$-dimensional Hjelmslev subspace $S$ we have

$$
\left|G_{S}\right| \cdot\left|S^{G}\right|=|G| \text {. }
$$

Since all orbits $S^{G}$ have the same size, the stabilizers $G_{S}$ have also the same size. For an arbitrary $g \in G$, we get from (4):

$$
\sum_{[S]^{(m-1)}} \sum_{L \in[S]^{(m-1)}} a(L) \rho\left(L^{g}\right)=0
$$


Let $g$ run over all elements of $G$. This implies

$$
\sum_{g \in G} \sum_{[S]^{(m-1)}} \sum_{L \in[S]^{(m-1)}} a(L) \rho\left(L^{g}\right)=\sum_{[S]^{(m-1)}} \sum_{L \in S} \sum_{g \in G} a(L) \rho\left(L^{g}\right)=\mathbf{0} .
$$

If $[L]^{(m-1)}=[M]^{(m-1)}$ the number of elements $g \in G$ for which $L^{g}=M$ is equal to the size of the stabilizer of $L$, i.e. $\left|G_{L}\right|=|G| /\left|L^{G}\right|$, and is hence constant for all Hjelmslev subspaces of the same dimension. Hence there exist coefficients $b\left([S]^{(m-1)}\right)$ such that

$$
\sum_{[S]^{(m-1)}} b\left([S]^{(m-1)}\right)\left(\sum_{L \in[S]^{(m-1)}} \rho(L)\right)=\mathbf{0} .
$$

Let the rows of the incidence matrix of $(s-1)$-dimensional vs. $(t-1)$-dimensional subspaces of $\operatorname{PHG}\left(R / R \theta(R / R \theta)^{n}\right)$ be $\boldsymbol{r}_{1}, \ldots, \boldsymbol{r}_{x}$. For a suitable ordering of the $(s-1)$-dimensional Hjelmslev subspaces of $\Omega$ and of the $(s-1)$-dimensional subspaces of $\mathrm{PG}\left(R / R \theta(R / R \theta)^{n}\right)$ we get

$$
\sum_{L \in\left[S_{i}\right]^{(m-1)}} \rho(L)=k\left(\boldsymbol{r}_{i} \otimes(\underbrace{1, \ldots, 1}_{v})\right) .
$$

Here $k$ denotes the number of ones in any column of the block $B$ defined above. This implies that

$$
\sum_{[S]^{(m-1)}} b\left([S]^{(m-1)}\right) \sum_{L \in[S]^{(m-1)}} \rho(L)=\sum_{i=1}^{x} b_{i} \cdot k(\boldsymbol{r}_{i} \otimes \underbrace{(1, \ldots, 1)}_{v})=0,
$$

where $b_{i}=b\left(\left[S_{i}\right]^{(m-1)}\right) k$. Hence

$$
\sum_{i=0}^{x} b_{i} \boldsymbol{r}_{i}=0
$$

a contradiction since by the induction hypothesis the rows $\boldsymbol{r}_{i}$ are linearly independent.

Now the proof of Theorem 5 is almost immediate.

Proof (Theorem 5) Let $t$ be the rank of the smallest free submodule of $R^{n}$ that contains a submodule of shape $\tau$. By $\boldsymbol{m}^{s} \preceq \tau \preceq \boldsymbol{m}^{n-s}$, we get $s \leq t \leq n-s$. Now we have

$$
M_{\boldsymbol{m}^{s}, \boldsymbol{m}^{t}}=\alpha M_{\boldsymbol{m}^{s}, \tau} M_{\tau, \boldsymbol{m}^{t}},
$$

where $\alpha$ is the number of submodules $U$ of shape $\tau$ with $S \subset U \subset T$, where $S$ and $T$ are fixed free submodules of ranks $s$ and $t$, respectively (hence $\alpha$ is a constant). Since $M_{\boldsymbol{m}^{s}, \boldsymbol{m}^{t}}$ is of full rank (by Theorem 7) then $M_{\boldsymbol{m}^{s}, \tau}$ is also of full rank by Sylvester's inequality.

\section{A counterexample and concluding remarks}

It might be tempting to conjecture that the matrix $M_{\sigma, \tau}(\Omega)$ is always of full rank, i.e. its rank is the smaller of the numbers $\left[\begin{array}{c}\boldsymbol{m}^{n} \\ \sigma\end{array}\right]_{q}$ and $\left[\begin{array}{c}\boldsymbol{m}^{n} \\ \tau\end{array}\right]_{q}$. Below we construct an example which demonstrates that this is not always true.

For the sake of simplicity we construct our example over the ring $\mathbb{Z}_{4}$, but it can be generalized to any chain ring. Take $R=\mathbb{Z}_{4}$ and consider the 3-dimensional Hjelmslev 
geometry $\Omega=\operatorname{PHG}\left({ }_{R} R^{4}\right)$. Set $\sigma=(2,1,0,0)$ and $\tau=(2,2,0,0)$. The shape $\sigma$ subspaces are line segments consisting of two points each; the shape $\tau$ subspaces are the lines of $\Omega$. Using Theorem 2 we find that the number of shape $\sigma$ subspaces is 420 while the number of shape $\tau$ subspaces is 560 (Theorem 2).

Let $S$ be a subspace of shape $\sigma$ in $\Omega$ and $T_{1}, T_{2}$-Hjelmslev subspaces of shape $\tau$ in $\Omega$ with $S \subset T_{1}, S \subset T_{2}$. Clearly $T_{1}$ and $T_{2}$ are Hjelmslev subspaces of minimal rank containing $S$. This implies that $T_{1}$ and $T_{2}$ are neighbors; otherwise the Hjelmslev subspace $T_{1} \cap T_{2}$ would contain $S$ which is a contradiction to the minimality of $T_{1}$ and $T_{2}$. This implies that there exists such ordering of the shape $\sigma$ and shape $\tau$ subspaces that $M_{\sigma, \tau}$ has diagonal block form with zero-blocks off the main diagonal. Each block has size $12 \times 16$ and there are 35 such blocks that correspond to the 35 lines in the factor geometry which happens to be $\mathrm{PG}(3,2)$.

Now the matrix $M_{\sigma, \tau}$ is of full rank if and only if each block is of full rank. Consider a single block $B$. It corresponds to a neighbor class of lines in $\Omega$. By Theorem 3, a block is isomorphic to a part of the point versus lines incidence matrix of $\operatorname{PG}(3,2)$. The rows are indexed by the twelve points not incident with a fixed line $\ell$ and the columns are indexed by the 16 lines skew to $\ell$. Now this matrix is not of full rank. To see this, let $\pi_{0}, \pi_{1}, \pi_{2}$ be the planes through $\ell$ and let the points in $\pi_{i}$ off $\ell$ be $P_{1}^{(i)}, \ldots, P_{4}^{(i)}, i=0,1,2$. Denote by $\rho(P)$ the row in $B$ indexed by the point $P$. Now it is easily checked that

$$
\sum_{j=1}^{4} \rho\left(P_{j}^{(0)}\right)=\sum_{j=1}^{4} \rho\left(P_{j}^{(1)}\right)=\sum_{j=1}^{4} \rho\left(P_{j}^{(2)}\right)=(\underbrace{1,1, \ldots, 1}_{16}) .
$$

This means that $B$ is not of full rank and hence $M_{\sigma, \tau}(\Omega)$ is also not of full rank.

It is clear that the same shapes considered in a higher dimensional space over the same ring will give again a matrix which is not of full rank.

By duality, Theorem 5 implies that in the case of shapes $\sigma$ and $\tau$ with $\boldsymbol{m}^{s} \preceq \sigma \preceq \boldsymbol{m}^{n-s}=\tau$, the matrix $M_{\sigma, \tau}(\Omega)$ is of full column rank. At present there is no reasonable conjecture about the shapes $\sigma \prec \boldsymbol{m}^{s} \preceq \tau \preceq \boldsymbol{m}^{n-s}$, for which the rank of $M_{\sigma, \tau}(\Omega)$ is maximal.

Acknowledgments The authors thank the anonymous referees for the numerous remarks and suggestions which improved the quality of this paper. This research is done within the COST Action IC-1004 "Random Network Coding and Designs over GF $(q)$ ". The first author has been supported by the Bulgarian NSF under Contract I-01/0003 and by Contract Nr. 100/19.04.2013 with the Science Research Fund of Sofia University. The research of the second author has been done as a part of the project "Finite geometries, coding theory and cryptography" between the Research Foundation-Flanders (FWO) and the Bulgarian Academy of Sciences.

\section{References}

1. Birkhoff G.: Subgroups of abelian groups. Proc. Lond. Math. Soc. 38(2), 385-401 (1934-1935).

2. Clark W.E., Drake D.A.: Finite chain rings. Abh. Math. Semin. der Univ. Hambg. 39, 147-153 (1974).

3. Honold T., Landjev I.: Projective Hjelmslev geometries. In: Proceedings of the International Workshop on Optimal Codes, Sozopol, pp. 97-115 (1998).

4. Honold T., Landjev I.: Linear codes over finite chain rings and projective Hjelmslev geometries, codes over rings. In: Sole P. (ed) (CNRS, France) Series on Coding Theory and Cryptology, vol. 6, pp. 60-123. World Scientific, Singapore (2009).

5. Honold T., Landjev I.: Codes over rings and ring geometries. In: Storme L., De Beule J. (eds .) Current Research Topics in Galois Geometries, pp. 161-186. NOVA Publishers, New York (2012).

6. Kantor W.M.: On incidence matrices of finite projective and affine spaces. Math. Z. 124, 315-318 (1972).

7. Landjev I., Vandendriessche P.: On the point-by-subspace incidence matrices of projective Hjelmslev spaces. Compt. Rend. Acad. Bulg. des Sci. (to appear) (2014). 
8. MacDonald I.G.: Symmetric Functions and Hall Polynomials, 2nd edn. Oxford University Press, Oxford (1995).

9. McDonald B.R.: Finite Rings with Identity. Marcel Dekker, New York (1974).

10. Nechaev A.A.: Finite principal ideal rings. Russ. Acad. Sci. Sbornik Math. 209, 364-382 (1973). 